\title{
Prognostic value of blood gas lactate levels among COVID-19 patients who visited to emergency department
}

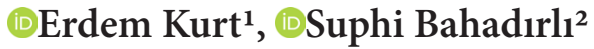 \\ ${ }^{1}$ Health Sciences University, İstanbul Training and Research Hospital, Deparmant of Emegency Medicine, İstanbul, Turkey \\ ${ }^{2}$ Beylikdüzü Public Hospital, Departmant of Emegency Medicine, İstanbul, Turkey
}

Cite this article as: Kurt E, Bahadırlı S. Prognostic value of blood gas lactate levels among COVID-19 patients who visited to emergency department. J Health Sci Med 2021; 4(4): 493-497.

\begin{abstract}
Introduction: Our aim in this study, to determine the relationship between the mortality status and the İntensive Care Unit (ICU) requirement of COVID-19 patients, with their blood gas lactate levels at the time of admission to the Emergency Medicine (ED).

Material and method: This study was planned as retrospectively, all COVID-19 patients over the age of 18 who were admitted to the ED, had oropharyngeal /nasopharyngeal swabs and hospitalized between April 1, 2020 and June 31, 2020 were included. The primary outcome was in-hospital mortality. The secondary outcome is to determine the relationship between lactate levels and the ICU requirement

Results: The study was completed with 265 patients after the inclusion and exclusion criteria were applied. The median (IQR) age of the patients was 43.0 (31.0-59.0), and 132 (49.8\%) were female. The comorbid diseases are; hypertension (HT), cardiovascular disease and diabetes mellitus (DM) were in the first three places with $23.0 \%, 17.0 \%, 9.1 \%$, respectively. The cut-off value of lactate to determined for ICU admissions was 2.92, and 4.25 for mortality. The AUC for ICU requirement was $0.718 \pm 0.038$ ( $95 \%$ CI, $0.644-0.792$ ), and AUC for mortality was $0.752 \pm 0.058$ ( $95 \% \mathrm{CI}, 0.637-0.867$ ), ( $<<0.001$ for both).
\end{abstract}

Conclusion: The COVID-19 pandemic has caused a serious mortality and morbidity problem worldwide. Our study found that hyperlactatemic COVID-19 patients had higher ICU requirement and mortality rates.

Keywords: COVID-19, lactate, mortality, emergency department

\section{INTRODUCTION}

In December 2019, a novel coronavirus, severe acute respiratory syndrome coronavirus 2 (SARS-CoV-2), was identified in Wuhan, China (1). The World Health Organization (WHO) named this infection as COVID-19. As a result of the rapid succession of cases all over the world, WHO announced a pandemic (2). The epidemic caused a serious mortality and morbidity problem worldwide, causing approximately 153 million cases and 3.2 million deaths as of May 1, 2021 (3).

Although most patients with COVID-19 are only mildly symptomatic, a proportion of patients can deteriorate significantly, causing multiple organ failure and resulting in death (4). The $32 \%$ of all positive patients require admission to the intensive care unit (ICU), and death may occur (5). Therefore, early diagnosis is important for the patients who have risk of serious illness and the potentially life-threatening conditions (6).
Lactate is a reversible product of anaerobic respiration in glucose metabolism and increases during ischemia. The presence of high serum lactate levels is strongly associated with morbidity and mortality in various critically ill populations (7).

Our aim in this study, to determine the relationship between the mortality status and the ICU requirement of COVID-19 patients, with their blood gas lactate levels at the time of admission to the emergency department (ED).

\section{MATERIAL AND METHOD}

In this study, patients who were admitted to ED of İstanbul Education and Training Hospital between April 1, 2020 and June 31, 2020 and were diagnosed with COVID-19 were examined retrospectively. Permission for this study 
was obtained by Health Sciences University, İstanbul Training and Research Hospital Clinical Researches Ethics Committee (Date: 30.04.2021, Decision No: 2816). All study procedures were conducted in compliance with the principles of the Declaration of Helsinki.

All COVID-19 patients over the age of 18 who were visited to the ED, had oropharyngeal /nasopharyngeal swabs and hospitalized between April 1,2020 and June 31, 2020 were included in this study. Patients whose reverse transcriptase polymerase chain reaction (RT-PCR) test results were negative and whose blood gas lactate value was not checked at the time of admission were excluded from the study. Additionally, patients who visited to the $\mathrm{ED}$ due to cardiac arrest and who were received inotropic support at the time of admission were not included in the study. Data were collected from electronic medical hospital records. The patients, all who included the study, were recorded in a form with their age, gender, blood gas lactate levels. Blood gas lactate levels were measured using the ABL800 FLEX blood gas analyser radiometer.

The primary outcome was in-hospital mortality. The secondary outcome is to determine the relationship between lactate levels and the ICU requirement. Outcomes were retrospectively assessed by reviewing of the hospital medical database.

\section{Statistical Analysis}

Categorical variables were presented as frequency and percentage. Continuous variables were tested for distribution using the Kolmogorov-Smirnov and Shapiro-Wilk tests. The asymmetrically distributed variables were expressed as the median interquartile range (25\%-75\%). All variables were compared for ICU admission and mortality outcomes using Pearson's chisquared, and Mann-Whitney $\mathrm{U}$ tests as appropriate. Receiver operating characteristic (ROC) analyses were performed to determine the predictive power of lactate in terms of both outcomes. The sensitivity, specificity, positive predictive value (PPV) and negative predictive value (NPV) of all cut points were calculated. The cutoff point that achieves the maximum Youden's index is referred to as the optimal cut-off. A 2-sided P-value of 0.05 was regarded as statistically significant. All data analyses were performed using SPSS version 23.0 software (SPSS Inc., Chicago, IL, USA).

\section{RESULTS}

The study was completed with 265 patients after the inclusion and exclusion criteria were applied. The median (IQR) age of the patients was 43.0 (31.0-59.0), and $132(49.8 \%)$ were female. When our study sample was examined in terms of comorbid diseases; hypertension (HT), cardiovascular disease and diabetes mellitus
(DM) were in the first three places with $23.0 \%, 17.0 \%$, $9.1 \%$, respectively. ICU admission was required in 68 (25.7\%) of all patients and $35(13.2 \%)$ died. The baseline characteristics of the patients were shown in Table 1.

\begin{tabular}{|lc|}
\hline \multicolumn{2}{|l|}{ Table 1. Baseline characteristics of 265 patients } \\
\hline Variables, median (IQR) or n(\%) & Total \\
\hline Age, years & $43.0(31.0-59.0)$ \\
Gender & $132(49.8)$ \\
Female & $133(50.2)$ \\
Male & \\
Comorbidities & $61(23.0)$ \\
HT & $24(9.1)$ \\
DM & $45(17.0)$ \\
Cardiovascular disease & $19(7.2)$ \\
Pulmonary disease & $20(7.5)$ \\
Cerebrovascular disease & $9(3.4)$ \\
Chronic renal failure & $15(5.7)$ \\
Malignancy & $105(39.6)$ \\
Any comorbidity & $1.88(1.13-3.30)$ \\
Lactate, mmol/L & \\
ICU & $197(74.3)$ \\
$(-)$ & $68(25.7)$ \\
$(+)$ & \\
Mortality & $230(86.8)$ \\
$(-)$ & $35(13.2)$ \\
$(+)$ & \\
\hline HT: Hypertension, DM: Diabetes mellitus, ICU: Intensive care unit \\
\hline
\end{tabular}

We compare age, gender and lactate variables in terms of ICU admission requirement and mortality. The median age in the group with ICU need was 57.0, and it was 37.0 in the other group. On the other hand, the median age of the patients who died was 61.0 years and those who recovered were 40.5 . These differences were statistically significant ( $\mathrm{p}<0.001$ for both). No significant difference was found in the gender variable in ICU need and mortality ( $\mathrm{p}=0.379, \mathrm{p}=0.837$; respectively). When we compare the lactate values, the lactate median was 3.12 in the group with ICU need and 1.58 in the group without ICU need. In terms of mortality, the difference was more dramatic. The lactate median of the patients who died was 4.90 , and that of those who recovered was 1.75. The differences between both outcome groups were statistically significant $(\mathrm{p}<0.001)$. The comparison of age, gender and lactate variables according to ICU admission and mortality outcomes were shown in Table 2.

ROC analysis was performed to determine the potency of lactate in predicting ICU admissions and mortality. The AUC for ICU requirement was $0.718 \pm 0.038(95 \%$ CI, 0.644 - 0.792), (Figure 1) and AUC for mortality was $0.752 \pm 0.058$ (95\% CI, 0.637 - 0.867), (Figure 2); (p $<0.001$ for both). 
Table 2. The comparison of age, gender and lactate variables according to ICU admission and mortality outcomes

\begin{tabular}{|lcccccc|}
\hline Variables & ICU (-) & ICU (+) & p & Mortality (-) & Mortality (+) & p \\
\hline Age & $37.0(29.0-51.0)$ & $57.0(47.0-74.3)$ & $<0.001^{*}$ & $40.5(31.0-55.0)$ & $61.0(48.5-73.5)$ & $<0.001^{*}$ \\
Gender & & $0.379^{* *}$ & & $114(49.6)$ & $18(51.4)$ & $0.837^{* *}$ \\
Female & $95(48.2)$ & $37(54.4)$ & & $116(50.4)$ & $17(48.6)$ & $4.90(2.89-7.22)$ \\
Male & $102(51.8)$ & $31(45.6)$ & & $1.75(1.11-2.74)$ & $<0.001^{*}$ \\
\hline Lactate & $1.58(1.07-2.46)$ & $3.12(2.00-3.89)$ & $<0.001^{*}$ & & \\
\hline ICU: Intensive care unit, ${ }^{*}$ Mann-Whitney U test, ${ }^{* *}$ Pearson's chi-squared test & & &
\end{tabular}

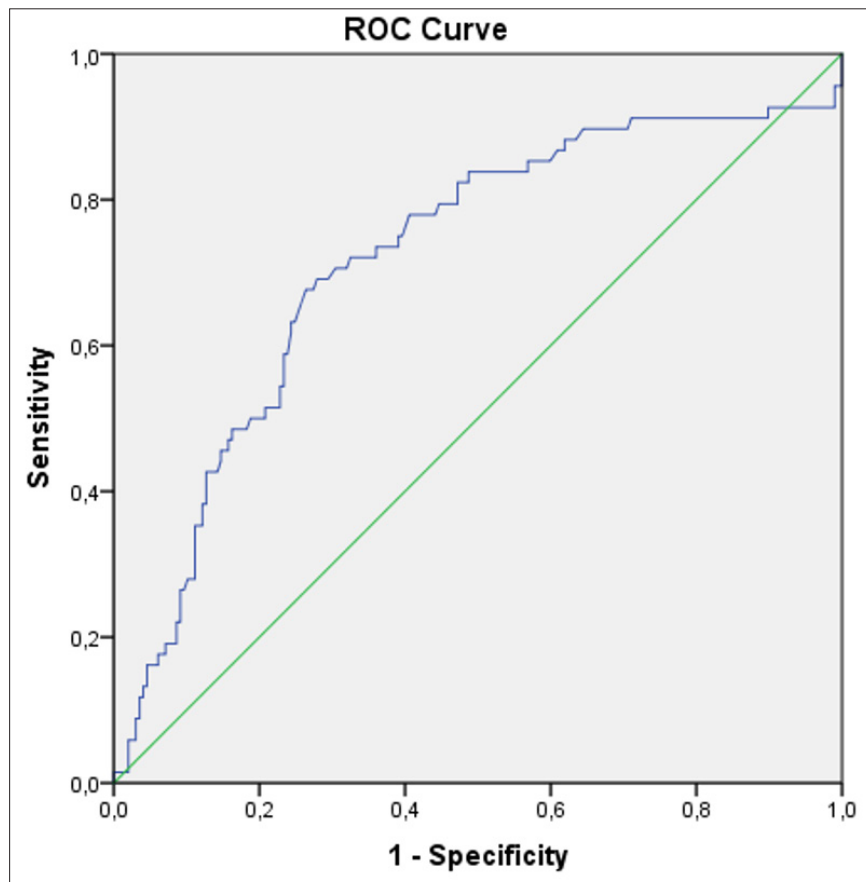

Diagonal segments are produced by ties.

Figure 1.The ROC curve of lactate for ICU admissions. AUC: $0.718 \pm 0.038$ (95\% CI, $0.644-0.792),(\mathrm{p}<0.001)$.

Then, the optimum cut-off points for the relevant outcomes were determined using the Youden's Index. The cut-off value determined for ICU admissions was 2.92, and 4.25 for mortality. The sensitivity, specificity, PPV, NPV, AUC and Youden's Index values of these cut-off points were shown in Table 3.

\section{DISCUSSION}

In this study, we aimed to determine the power of blood gas lactate levels at the time of ED admission in predicting in-hospital mortality and ICU requirement, in patients diagnosed with COVID-19 and hospitalized. In this cohort, we concluded that increased lactate levels could be useful in predicting in-hospital mortality and ICU requirement.

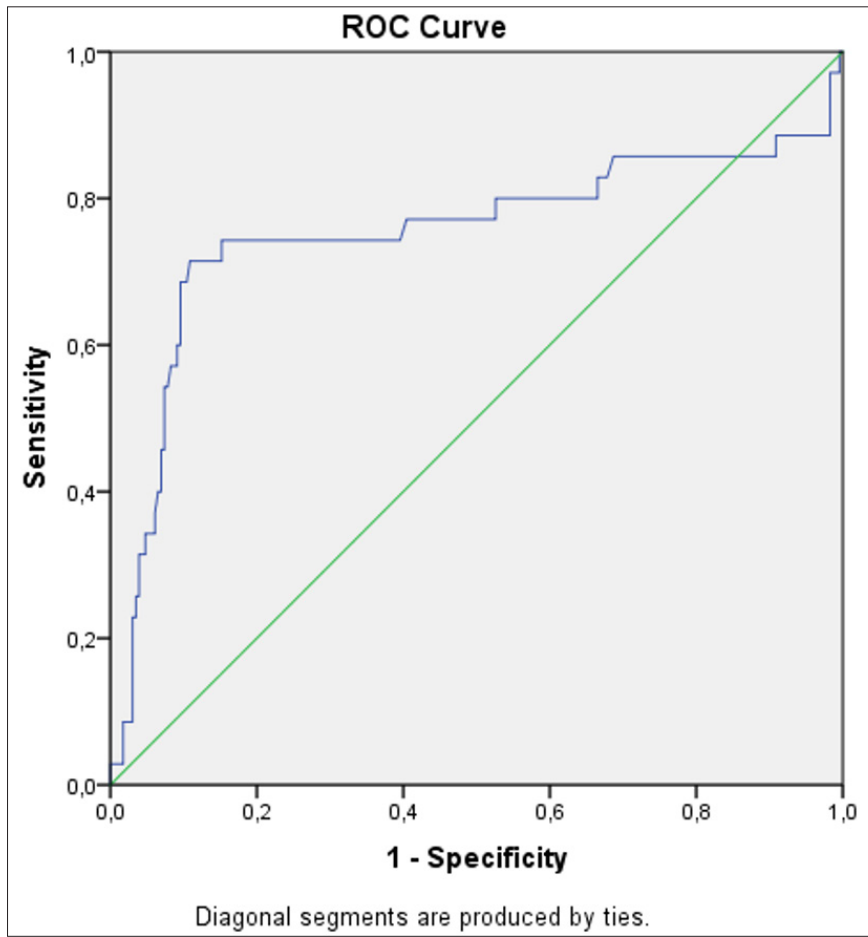

Figure 2.The ROC curve of lactate for mortality. AUC: $0.752 \pm 0.058(95 \% \mathrm{CI}, 0.637-0.867),(\mathrm{p}<0.001)$

In a study evaluating the clinical course and risk factors on hospitalized COVID-19 patients, ICU requirement was $26 \%$ and the mortality prevalence among the patients admitted to the ICU was $22 \%$. (8). Although our study is consistent with the literature, the relatively low mortality rates can be linked to the low average age of our study population.

In this study, it was found that the average age of the patients with a mortal course and ICU requirement was high. There are similar studies in the literature examining the effect of age on poor outcome. In a retrospective study of 710 patients, the ICU requirement and 28-day mortality were examined, and it was emphasized that the outcome was poor in the elderly population (9).

Table 3. Optimum cut-off points ${ }^{\star}$ of Lactate in predicting ICU admissions and in-hospital mortality

\begin{tabular}{lcccccccc} 
& Cut-off point & Sens (\%) & Spec (\%) & PPV (\%) & NPV (\%) & AUC & Youden's Index \\
\hline ICU admissions & 2.92 & 67.65 & 74.62 & 47.92 & 86.98 & 0.718 & 0.423 \\
Mortality & 4.25 & 71.43 & 89.13 & 50.00 & 95.35 & 0.752 & 0.606 \\
\hline$*$
\end{tabular}

* Cut-off points with the highest Youden's index value were shown, ICU: Intensive care unit, Sens: Sensitivity, Spec: Specificity, PPV: Positive predictive value, NPV: Negative predictive value AUC: Area under the curve 
The ICU requirement and the mortality are more common in patients with COVID-19 in the presence of some chronic diseases (10). For this purpose, studies examining the comorbidity of patients are available in the literature. In a study conducted in China on COVID-19 patients, it was reported that the most common comorbidity was hypertension (16.9\%), followed by diabetes $(8.2 \%)$, and $8.2 \%$ of patients with two or more comorbidities (11). In a retrospective study conducted in Wuhan, it was reported that hypertension was the most common comorbidity, followed by diabetes and coronary heart disease (12). The most common comorbidity in our study is hypertension, followed by cardiovascular diseases and diabetes mellitus. These results were found to be suitable with the literature.

Lactate, which is produced in almost all considerable tissues (skeletal muscle, brain, erythrocytes, and kidneys) even under conditions where oxygen is sufficient, is kept below $1 \mathrm{mmol} / \mathrm{L}$ in both arterial and venous blood, especially by liver metabolism and recycling into the pyruvate. The lactate levels in the blood begin to increase, if insufficient oxygen is provided to the tissues or the oxygen demand of the tissues increases (13). Lactate is the reversible product of anaerobic respiration in glucose metabolism and it increases during ischemia. In the case of hypoxia, the formation of NAD (Nicotinamide Adenine Dinucleotide) decreases, thus the NADH/NAD ratio increases and the lactate level increases (14).

While the hyperlactatemia is defined as a persistent, mild to moderate $(2-4 \mathrm{mmol} / \mathrm{L})$ increase in blood lactate concentration without metabolic acidosis, the lactic acidosis is characterized with the blood lactate levels (usually> $5 \mathrm{mmol} / \mathrm{L}$ ), which consistently increased by metabolic acidosis (15).

In this study, we found that high lactate levels observed in COVID-19 patients were significant in predicting ICU requirement and in-hospital mortality. The prognostic ability of lactate has been proven as independent of organ failure and shock, in patients with severe sepsis (7). Previous studies have shown that lactate can be used as an early identifier of organ failure and latent shock before any detectable changes in a patient's vital signs ocur (16). In a study conducted in patients admitted to the emergency department with a diagnosis of pneumonia, it is stated that lactate is more valuable than CURB-65 in predicting mortality, hospitalization, and ICU requirement (17). In another prospective study conducted on sepsis patients, it is stated that the high lactate level is effective in determining the severity of sepsis (18).

As with any retrospective study, there are some limitations in this study. The sample size of this single centre study was relatively small; therefore, more studies with a larger sample size are needed to confirm these results.

\section{CONCLUSION}

The COVID-19 pandemic has caused a serious mortality and morbidity problem worldwide. Our study found that hyperlactatemic COVID-19 patients had higher ICU requirement and mortality rates. Prospective studies with larger sample sizes are necessary to adequately assess the relationship between lactate and mortality in COVID-19 patients.

\section{ETHICAL DECLARATIONS}

Ethics Committee Approval: Permission for this study was obtained by Health Sciences University, İstanbul Training and Research Hospital Clinical Researches Ethics Committee (Date: 30.04.2021, Decision No: 2816).

Informed Consent: Because the study was designed retrospectively, no written informed consent form was obtained from patients.

\section{Referee Evaluation Process: Externally peer-reviewed.}

Conflict of Interest Statement: The author(s) declared no potential conflicts of interest with respect to the research, authorship, and/or publication of this article.

Financial Disclosure: The authors have not declared a specific grant for this research from any funding agency in the public, commercial or not-for-profit sectors.

Author Contributions: All of the authors declare that they have all participated in the design, execution, and analysis of the paper, and that they have approved the final version.

\section{REFERENCES}

1. Gürbüz T, Gökmen O, Kaptan G, et al. Investigating anxiety, depression and obsessive-compulsive disorders among the pregnant women during Covid-19 pandemic. J Health Sci Med 2021; 4: 7-12.

2. Seyhan AU, Doganay F, Yilmaz E, Topal NP, Ak R. Investigation of QT prolongation with hydroxychloroquine and azithromycin for the treatment of COVID-19. J Coll Physicians Surg Pak 2020; 30: 153-7.

3. COVID Live Update: $157,636,912$ Cases and 3,286,500 Deaths from the Coronavirus - Worldometer. https://www.worldometers. info/coronavirus

4. Huang C, Wang Y, Li X, et al. Clinical features of patients infected with 2019 novel coronavirus in Wuhan, China. The Lancet 2020; 395: 497-506.

5. Phua J, Weng L, Ling L, et al. Asian Critical Care Clinical Trials Group. Intensive care management of coronavirus disease 2019 (COVID-19): challenges and recommendations. The Lancet Res Med 2020; 8: 506-17.

6. Doğanay F, Elkonca F, Seyhan AU, Yılmaz E, Batırel A, Ak R. Shock index as a predictor of mortality among the Covid-19 patients. Am J Emerg Med 2021; 40: 106-9.

7. Mikkelsen ME, Miltiades AN, Gaieski DF, et al. Serum lactate is associated with mortality in severe sepsis independent of organ failure and shock.Crit care Med 2009; 37: 1670-7.

8. Zhou F, Yu T, Du R, et al. Clinical course and risk factors for mortality of adult inpatients with COVID-19 in Wuhan, China: a retrospective cohort study. The Lancet 2020; 395: 1054-62. 
9. Yang X, Yu Y, Xu J, et al. Clinical course and outcomes of critically ill patients with SARS-CoV-2 pneumonia in Wuhan, China: a single-centered, retrospective, observational study. Lancet Respir Med 2020; 8: 475-81.

10. Sanyaolu A, Okorie C, Marinkovic A, et al. Comorbidity and its impact on patients with COVID-19. SN Compr Clin Med 2020; 1-8.

11. Guan WJ, Liang WH, Zhao Y, et al. Comorbidity and its impact on 1590 patients with COVID-19 in China: a nationwide analysis. Eur Respir J 2020: 55-5.

12.Zhou F, Yu T, Du R, et al. Clinical course and risk factors for mortality of adult inpatients with COVID-19 in Wuhan, China: a retrospective cohort study. The Lancet 2020; 395: 1054-62.

13. İbrahim A, Bayramoglu B, Hokenek NM, et al. Lactate clearance during the first 2 hours after hospital admission: A useful biomarker for predicting 30-day mortality in patients with diabetic ketoacidosis. Int J Clin Pract 2021; e14204.

14. Hökenek NM, Seyhan AU, Erdoğan MÖ, Tekyol D, Yllmaz E, Korkut S. Evaluation of blood gas analysis as a mortality predictor. S Clin Ist Eur 2019; 30-3.

15.Sakal C, Ak R, Taşçı A, Deniz KE, Akoğlu EU, Cimilli OT. Admission blood lactate levels of patients diagnosed with cerebrovascular disease effects on short and long-term mortality risk. Int J Clin Prac 2021; e14161.

16. Juneja D, Singh O, Dang R. Admission hyperlactatemia: causes, incidence, and impact on outcome of patients admitted in a general medical intensive care unit. J Crit Care 2011; 26: 316-20.

17. Chen YX, Li CS. Lactate on emergency department arrival as a predictor of mortality and site-of-care in pneumonia patients: a cohort study. Thorax 2015; 70: 404-10.

18. Contenti J, Corraze H, Lemoël F, Levraut J. Effectiveness of arterial, venous, and capillary blood lactate as a sepsis triage tool in ED patients. Am J Emerg Med 2015; 33: 167-72. 